\title{
Medikamentöse Therapien auf dem Prüfstand
}

Fragestellung: Welches ist die effektivste Strategie zur medikamentösen Behandlung akuter Rückenschmerzen?

Hintergrund: Nach Leitlinienempfehlungen sollen akute Rückenschmerzen über einen begrenzten Zeitraum mit nicht steroidalen Antirheumatika (NSAR) und/oder Muskelrelaxanzien behandelt werden. Zum Teil werden auch Opioide empfohlen. Ein guter Vergleich zwischen den Kombinationen NSAR plus Muskelrelaxanzien und Opioide plus Paracetamol fehlte bisher.

Patienten und Methodik: In die Studie wurden Patienten mit akuten Rückenschmerzen aufgenommen, die sich in einer Notfallaufnahme vorstellten. $\mathrm{Pa}$ tienten mit radikulären

Friedman BW, Dym AA, Davitt $M$ et al. Naproxen with cyclobenzaprine, oxycodone/acetaminophen, or placebo for treating acute low back pain: A randomized clinical trial. JAMA 2015; 314: $1572-80$
Schmerzen wurden ausgeschlossen. Die Patienten mussten im Roland-MorrisDisability Questionnaire (RMDQ), der auf einer Skala von 0 bis 24 Punkten Schmerz und Einschränkung bei Rückenschmerzen misst, bei Studieneinschluss einen Wert von mindestens 5 Punkten aufweisen. Alle Patienten erhielten als Basistherapie zweimal täglich $500 \mathrm{mg}$ Naproxen. Zusätzlich bekamen sie täglich entweder Placebo, ein Muskelrelaxans oder Oxycodon in Kombination mit Paracetamol. Der primäre Endpunkt war die Reduktion der Rückenschmerzen im RMDQ nach einer Woche.

Ergebnisse: Die 323 Patienten waren im Mittel 39 Jahre alt und litten zum Zeitpunkt der Randomisierung seit 48 Stunden unter Rückenschmerzen. Der Median des RMDQ-Scores betrug 20 Punkte. Etwa die Hälfte der Patienten hatte bereits früher unter Rückenschmerzen gelitten. Mit Placebo besserten sich die Rückenschmerzen um 9,8 Punkte, mit dem Muskelrelaxans um 10,1 Punkte und mit dem Opioid plus Paracetamol um 11,1 Punkte. Die Gruppenunterschiede waren statistisch nicht signifikant.

Schlussfolgerungen: Die zusätzliche Gabe von Muskelrelaxanzien oder Opioiden plus Paracetamol ist einer Monotherapie mit Naproxen bei akuten Rückenschmerzen nicht überlegen.

\section{- Kommentar von Hans-Christoph Diener, Essen}

\section{Zunächst reicht ein ausreichend dosiertes NSAR}

Die Ergebnisse dieser Studie haben Konsequenzen für Patienten, die mit akuten Rückenschmerzen in einer neurologischen Notaufnahme vorgestellt werden. Offenbar reicht es zunächst aus, ein ausreichend dosiertes NSAR zu geben. Die zusätzliche Gabe von Muskelrelaxanzien und einer Kombina- tion eines Opioids mit Paracetamol erbringt offenbar keinen zusätzlichen Nutzen im Hinblick auf die Reduktion der Rückenschmerzen nach einer Woche. Patienten mit radikulären Schmerzen waren allerdings von der Teilnahme an dieser Studie ausgeschlossen.

\section{KVT ebenso effektiv wie Lichttherapie}

Fragestellung: Die Studie verglich die Effektivität von kognitiver Verhaltenstherapie (KVT) und Lichttherapie (LT) bei Patienten mit saisonaler Depression bezüglich Outcome und Remissionsrate.

Hintergrund: Die LT stellte bisher die Therapie der ersten Wahl bei saisonaler Depression dar, jedoch werden alternative Behandlungsmethoden für therapieresistente Patienten gefordert. Erste Studien zeigten vielversprechende Ergebnisse mittels einer speziell für saisonale affektive Störungen (SAD) konzipierten KVT. Eine vorausgehende randomisierte kontrollierte Studie [1] zeigte eine ähnliche Effektivität für LT und KVT. Darauf basierend erfolgte die vorliegende Studie mit dem Ziel einer höheren
Validität sowie eines zusätzlichen zweijährigen Follow-up nach einer ersten Therapieepisode.

Patienten und Methodik: Im Rahmen einer randomisierten, prospektiven, kontrollierten, mehrfach verblindeten Studie wurden an der Universität Vermont 1.495 potenzielle Teilnehmer durch lokale Medienwerbung erreicht. Nach einem TelefonScreening wurden 485 Probanden zum SCID-Interview (Structured Clinical Interview for DSM-IV Axis I Disorders) eingeladen. Davon erfüllten 240 Probanden die Studienkriterien und wurden zur Testung des Schweregrads der saisonalen Depression bei Baseline mittels SIGH-SAD (Structured Interview Guide for Hamilton Depression Rating Scale - Seasonal Affective 